\title{
A NEW METHOD FOR RECONSTRUCTING BIBLICAL SCROLLS ${ }^{1}$
}

\author{
Edward D. Herbert
}

The Qumran biblical scrolls are of crucial importance for developing our understanding of the textual diversity that existed around the turn of the era, and for their text-critical contributions. Biblical scholars have generally depended primarily upon common sense and general scholarly judgement in reconstructing such scrolls. Chapter 1 advances a range of scientifically-based tools for reconstructing biblical scrolls, which will be summarised below under six headings. In the remainder of the thesis, these tools are applied to the reconstruction of the $4 \mathrm{QSam}{ }^{\mathrm{a}}$ fragments of 2 Samuel.

Development 1: Expected Widths. Amounts of text are traditionally measured by counting characters. This constitutes an approximation of the space occupied that takes no account of the widely varying widths of the letters of the Hebrew alphabet. In 1QIsa, for example, the largest letter (ש) is over 2.5 times the size of a $T$ and over 3.5 times the size of a final $\}$. The proposed new measure takes full account of the differing letter widths. It entails calculating, for the scroll in question, the average width of each letter of the alphabet, and, based on this, an 'expected width' for each proposed reconstruction is calculated, which is the sum of the average letter widths of its constituent letters. Thus the expected width of $\$$ is the sum of the average widths of an $\aleph, a \zeta$, and the following space.

Development 2: Vertical Dividers. A closely linked development is the construction of vertical dividers, which are vertical lines which link points that are directly above each other on an extant fragment. Expected widths are therefore

\footnotetext{
${ }^{1}$ Edward D. Herbert, A New Method for Reconstructing Biblical Scrolls, and its Application to the Reconstruction of $4 \mathrm{QSam}$ (Unpublished Ph.D. Thesis, University of Cambridge, 1995); Supervisor: Dr. G.I. Davies.
} 
calculated, not just for complete lines, but for sections, which represent a part of a line that is bounded on both sides by a vertical divider, or by a vertical divider and the right margin. The expectation here is that the amount of material between two given dividers would be similar for each line. This not only places the focus on shorter sections of a line, but also removes the effect of the 'ragged' left margin from all but the left-hand section.

Development 3: Table of Critical \% Deviations. The question now arises as to how much the expected length of a section has to differ from the average of the corresponding sections of neighbouring lines before one has to conclude that the reconstruction is implausible. Where the difference is substantial (say, $50 \mathrm{~mm}$ against a range of $39-42 \mathrm{~mm}$ for corresponding sections of neighbouring lines), it is reasonably clear that the proposed reconstruction is incorrect, but, there again, this could probably have been ascertained even by using character counts. On the other hand, where the difference is less clear, should the proposed reconstruction be rejected or not? If the reconstructed section were, say, $47 \mathrm{~mm}$, is that viable? Or what about if it is $44 \mathrm{~mm}$ ? The thesis shows how the extant fragments of a scroll can be used to construct a table showing, for each section size range, the level of deviation which could occur by chance on only $5 \%$ of occasions, and the (obviously greater) level of deviation which could occur by chance on only $1 \%$ of occasions. In assessing whether a given reconstruction is viable, the deviation of its expected width from the average of comparable sections from neighbouring lines is calculated and compared to the table to see whether it exceeds the $5 \%$ or $1 \%$ thresholds ('significance levels'). If it does exceed one of these, then 'substantial suspicion' or 'reasonable confidence' respectively is raised that the reconstruction is incorrect.

Development 4: Margin Identification. The positions of the right and left margins represent valuable data for reconstruction. A known right margin effectively creates an extra vertical divider, and a known left margin reduces the uncertainty arising from its ragged nature. The primary means of locating non-extant margins is by seeking a margin position 
for which the right-hand section's expected widths are similar. Margin positions may also be deduced from the presence of paragraph breaks, represented by vacats at the beginning, middle or end of a line. A large shortfall of material (say, over $50 \mathrm{~mm}$ ) is generally too large to be an indented line or a midline gap, and so is likely to be a line foreshortening, which in turn suggests the margin position. In a scroll such as $4 Q S a m a$, where mid-line gaps are very rare, the presence of a paragraph break can be even more informative. Where the margin position suggested by paragraphing considerations coincides with that suggested by consistent widths of the right-hand section, its position can be accepted with confidence.

Development 5: Scribal Margin Policy. In most Qumran texts the left margin is not left-justified. How, then, did the scribe decide when to write a new word on a new line? Tests on five substantially extant scrolls (1QIsa, 1QM, 11QT, 1QpHab, and $1 Q S$ ) showed that none of these scribes adhered to the stipulations in the Talmudic minor Tractate Soperim. In 1QM (and 4 QSam ${ }^{\mathrm{a}}$ ), the best model of scribal margin policy is that the scribe sought to minimise the distance between the end of the last word of the line and the ruled left margin. So if a word finishes $6 \mathrm{~mm}$ before the ruled margin, and the next available word is $11 \mathrm{~mm}$, this next word would be placed at the end of this line, since it would reduce the gap between end of line and margin from $6 \mathrm{~mm}$ to $5 \mathrm{~mm}$. In other scrolls, an amendment to this model was required, whereby the scribe sought to minimise the distance between the end of the last word of a line and a notional margin, usually to the left of the ruled margin. This model inaccurately predicts the last word of a line in only $3.5 \%$ of cases, and with certain sophistications, is inaccurate in less than $1 \%$ of cases. The proposed reconstruction method incorporates this model, and thus significantly reduces the problems introduced by non-extant left margins.

Development 6: Column Analysis. A variety of techniques have been assembled to assist in estimating the locations of the breaks between columns. These are particularly useful for scrolls (such as 4 QSam ${ }^{a}$ ) for which the column widths vary significantly. It can also sometimes enable major pluses or minuses to be inferred in the non-extant parts of a column. 
Two alternative approaches to developments 1-3 have previously been advanced by scholars. First, Carol Newsom has adapted the mere usage of character counts by counting the shortest letters as half characters. Tests on five substantially extant scrolls demonstrated that Newsom's 'corrected letter space' method indeed represents a significant improvement over uncorrected character counts, but only a third of the improvement that arises from the use of expected widths. The second approach is the computer-assisted approach to reconstruction described by Lange, which involves cut-andpasting digitised copies of actual extant characters (or character groups) from elsewhere in the scroll. This method is attractive because it takes account of the variations in the widths of the different letters of the Hebrew alphabet, but should we accept a reconstruction where the pasted letters use up $15 \%$ more space than is available in the gap? Or $10 \%$ ? Or $5 \%$ ? The expected width method, which is based upon numerical calculations is, by contrast, ideally suited to the statistical assessment of the degree of deviation.

The focus then moves to the important but fragmentary biblical scroll, 4QSama. Chapter 2 presents 62 new fragment identifications and an analysis of the character of the scroll (orthography, margin and paragraphing policies, consistency of writing size, etc.), as the basis for the detailed reconstruction of the fragments of 2 Samuel in chapter 3. This reconstruction aims to identify all deviations of the scroll from the Masoretic Text, together with an analysis of the support of the other key Hebrew and Greek witnesses. The resulting list of deviations is over $55 \%$ greater than all previously identified deviations put together (over $30 \%$ if only those directly arising from developments in method are counted), demonstrating the effectiveness of the proposed method, and providing an improved foundation for projected analyses of the scroll's textual relationship to Chronicles and the Septuagint.

The thesis concludes with a brief consideration of the application of the method to other (often more fragmentary) biblical scrolls, and to non-biblical scrolls. 